\title{
Effect of barium titanate seed particles on the sintering and lattice parameters in $\mathrm{PbMg} \mathrm{Mb}_{1 / 3} \mathrm{Nb}_{2 / 3} \mathrm{O}_{3}$ ceramics
}

\author{
P.D. Spagnol, ${ }^{\text {a) }}$ M.A. Zaghete, and C.O. Paiva-Santos \\ Chemistry Institute, Physics-Chemistry Department, UNESP PO Box 355, \\ Araraquara (SP), Brazil, 14801-970
}

A.V.C. Andrade

Universidade Estadual de Ponta Grossa. Physics Department, Ponta Grossa (PR), Brazil, 84.030-000

A.A. Cavalheiro

Chemistry Institute, Physics-Chemistry Department, UNESP PO Box 355, Araraquara (SP), Brazil, 14801-970

S.M. Tebcherani

Universidade Estadual de Ponta Grossa. Chemistry Department, Ponta Grossa (PR), Brazil, 84.030-000

J.A. Varela

Chemistry Institute, Physics-Chemistry Department, UNESP PO Box 355, Araraquara (SP), Brazil, 14801-970

(Received 4 May 2001; accepted 28 December 2001)

\begin{abstract}
$\mathrm{PbMg}_{1 / 3} \mathrm{Nb}_{2 / 3} \mathrm{O}_{3}$ (PMN) powder was prepared by citrate organic solution, and barium titanate (BT) seed particles were added to encourage the perovskite phase formation. Sintering was followed using the constant heating rate mode of a dilatometer, and it was observed that the seed concentration affected the PMN shrinkage rate and crystal structure. The study of the lattice parameters of the samples after the sintering process indicates that the diffusion of the titanium and of the barium inside perovskite and pyrochlore PMN phases occurs. Moreover, this substitution provoked a decrease of the lattice parameters as showed by the Rietveld refinement.
\end{abstract}

\section{INTRODUCTION}

Lead-based ferroelectrics are increasingly being used as capacitors, tunable transducers, actuators, and memory devices. ${ }^{1}$ These materials are also used as micropositioners or actuators in dot-matrix printers and videotape heads because of their large electrostrictive strains. Most of these materials are lead magnesium niobate $\left(\mathrm{PbMg}_{1 / 3} \mathrm{Nb}_{2 / 3} \mathrm{O}_{3}\right.$; $\mathrm{PMN})$ dielectrics that have a high dielectric constant $(\mathrm{k}>$ $15,000)$ and low sintering temperature. ${ }^{1}$

Depending on the processing conditions, a second phase that has the pyrochlore structure may be present, which reduces the dielectric constant of the material. ${ }^{2}$ It is difficult to prepare PMN phase-pure perovskite (pyrochlore free) ferroelectrics via the conventional solid-state method. Methods such as coprecipitation, sol-gel, ${ }^{3,4}$ microencapsulation, molten salt, organic solution of citrates, ${ }^{5}$ and the two-step columbite method ${ }^{6,7}$ have been

a) Address all correspondence to this author.

e-mail: priscilaspagnol@yahoo.com used for processing relaxors with reduced amounts of pyrochlore phase. The main problems in the processing of lead-based ferroelectrics are (i) formation of a lowdielectric phase (such as pyrochlore), (ii) $\mathrm{PbO}$ volatilization, and (iii) irreproducible dielectric property. ${ }^{1}$

It is known that ceramic materials are transformed via a nucleation and growth process, often requiring high temperatures to surmount the large energy barriers of the nucleation and growth the stable phase. Consequently, these energy barriers frequently determine the calcination conditions, and therefore the characteristics such as particle size, morphology, and degree of aggregation of the precursor powder. Therefore, controlled nucleation and growth processes can play a significant role in processing control of advanced ceramics. ${ }^{8}$

The objective of the present study is to verify the influence of barium titanate (BT) seed concentration on sintering behavior, the lattice parameter of PMN, and the amount of perovskite phase. The effectiveness of the BT seed addition on the perovskite phase formation is well known, but the way the seed acts is not clear. 


\section{EXPERIMENTAL PROCEDURE}

PMN ceramic powder was prepared according to the method developed by Pechini. ${ }^{9}$ This process involves the ability of certain weak acids to form polybasic acid chelates with various cations from elements such as $\mathrm{Ti}$, $\mathrm{Zr}, \mathrm{Cr}, \mathrm{Mn}, \mathrm{Ba}, \mathrm{La}, \mathrm{Pb}, \mathrm{Nb}, \mathrm{Mg}$, etc. These chelates can undergo polyesterification when heated in polyhydroxyl alcohol to form a polymeric glass with cations uniformly distributed throughout. Thus the glass retains homogeneity on the atomic scale and may be calcined at a low temperatures to yield fine particulate oxides whose chemistry has been precisely controlled. In this process, niobium citrate solution at $\mathrm{pH}=7$ was prepared, and then ethylene glycol was added at a molar ratio of 1:3:12 (metal:citric acid:ethylene glycol). An aqueous solution of stoichiometric magnesium carbonate and lead acetate were added very slowly to the niobium citrate solution at a temperature of $90{ }^{\circ} \mathrm{C}$. Magnetic stirring agitated the mixture until complete dissolution. The obtained solution was concentrated by water evaporation, and at this point the $\mathrm{BaTiO}_{3}$ (BT) seed particles were added (1, 3, 5, and 10 mass \%) under magnetic stirring agitation. The viscosity was high enough to keep the seed particles dispersed in the solution, avoiding to decant. The temperature was then increased to about $130-140{ }^{\circ} \mathrm{C}$ to initiate polyestherification and water removal. Soon after, the dried solution was heat-treated in air at $200-350{ }^{\circ} \mathrm{C}$ in a box furnace with injection of air to eliminate the organic material. The resulting porous solid material was milled in a mortar.

The seed particles were obtained by the hydrothermal synthesis method ${ }^{10}$ and characterized by $\mathrm{x}$-ray diffraction (XRD; Rigaku-Rotaflex, Japan) and by particle-size distribution (measured using a centrifugal particle-size analyser, Horiba Kapa 900, Japan). Before they were added to the solution, the seed particles were deagglomerated in ethylene glycol using a high frequency ultrasound probe.

The powder was calcined at $800{ }^{\circ} \mathrm{C}$ for $2 \mathrm{~h}$ and characterized by XRD. The obtained powders was milled in an atritor mill (SZEGVARI, Hungary) at $400 \mathrm{rpm}$ for 30 min in acetone with zirconia balls. The powder was then dried and screened in a sieve with mesh 80. After that, the powders were pressed uniaxialy $(10 \mathrm{MPa})$ and isostaticaly $(210 \mathrm{MPa})$ in the form of pellets. A horizontal dilatometer (NETZSCH Model 402E, Germany) was used to study the sintering behavior of the pellets.

The pellets were also sintered at $1200{ }^{\circ} \mathrm{C}$ for $3 \mathrm{~h}$ in a closed system with addition of a sacrifice powder rich in lead to limit the $\mathrm{PbO}$ loss in the samples. These sintered compacts were characterized by the Rietveld method $^{11}$ with $\mathrm{x}$-ray diffraction data. The program used was the DBWS-9807a, which is an upgrade of the program DBWS-9411. ${ }^{12}$ The pseudo-Voigt function was used as a profile function.
The nomenclature used for the samples prepared in this work was pure PMN (PMNP) and PMN with addition of $1 \mathrm{wt} \%$ (PMN1BT), $3 \mathrm{wt} \%$ (PMN3BT), $5 \mathrm{wt} \%$ (PMN5BT), and $10 \mathrm{wt} \%$ (PMN10BT) BT.

\section{RESULTS AND DISCUSSION}

As commented by Mcardle and Messing, ${ }^{13}$ it is of extreme importance that the particles used as seeds should possess lattice parameters similar to the phase that one wants to obtain to assist in the nucleation process. In this case, BT is isostructural with PMN and the lattice parameters differ by approximately $1 \%$. Another parameter that should be considered is that the particle seeds should possess a narrow distribution of particle size to favor the formation of homogeneous PMN particles. X-ray diffractometry of the BT seed particles indicated a cubic crystal structure at room temperature, which is consistent with the literature reports that the cubic-tetragonal ferroic transition in BT is inhibited in the nanometric size range. ${ }^{19}$ Particle-size distribution by sedimentation process was a narrow distribution with a mean particle size of $0.6 \mu \mathrm{m}$ (Fig. 1), and transmission electron microscopy (TEM) of the BT seeds (Fig. 2) showed the morphology of the BT particles.

These powders were characterized by XRD to identify the phases formed during the calcination process at $800{ }^{\circ} \mathrm{C}$ for $2 \mathrm{~h}$, and the quantification of perovskite phase versus BT seed is shown in Fig. 3. The following relation was used to calculate qualitatively the weight fraction of the perovskite phase.

$$
\text { Fraction of perovskite }=\frac{I_{\text {pero(110) }}}{I_{\text {pero }(110)}+I_{\text {pyro(22) }}} \times 100
$$

We can notice that the addition of BT seed increases the fraction of perovskite phase in relation to the unseeded sample because it contributes to nucleus formation of a structure similar to the perovskite phase.

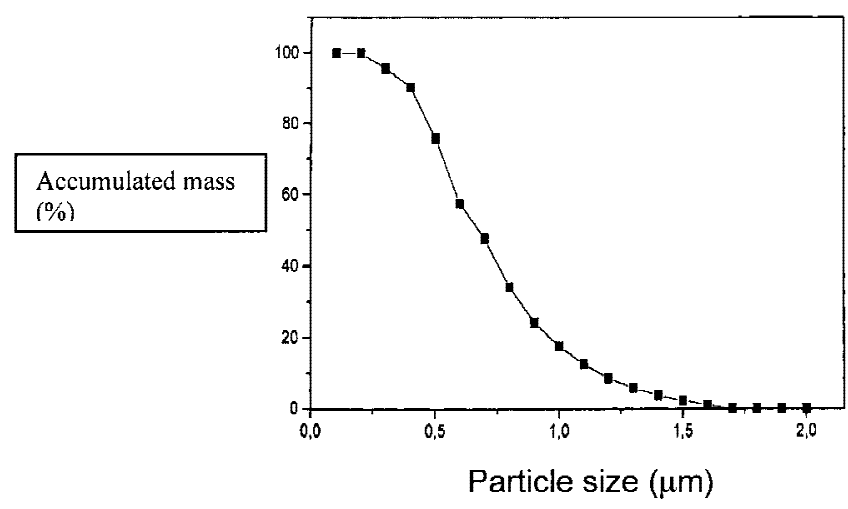

FIG. 1. Particle size distribution by sedimentation process for $\mathrm{BaTiO}_{3}$. 
Another important observation is that, after $3 \mathrm{wt} \% \mathrm{BT}$, no significant difference in the improvement of perovskite phase was verified. This result is in agreement with that observed by Narendar and Messing. ${ }^{20}$ The increase in the fraction of perovskite phase can be justified by the presence of seed nucleus, which possesses a lattice parameter very similar to the desired perovskite phase, only $1 \%$ misfit, providing patterns of growth favoring the

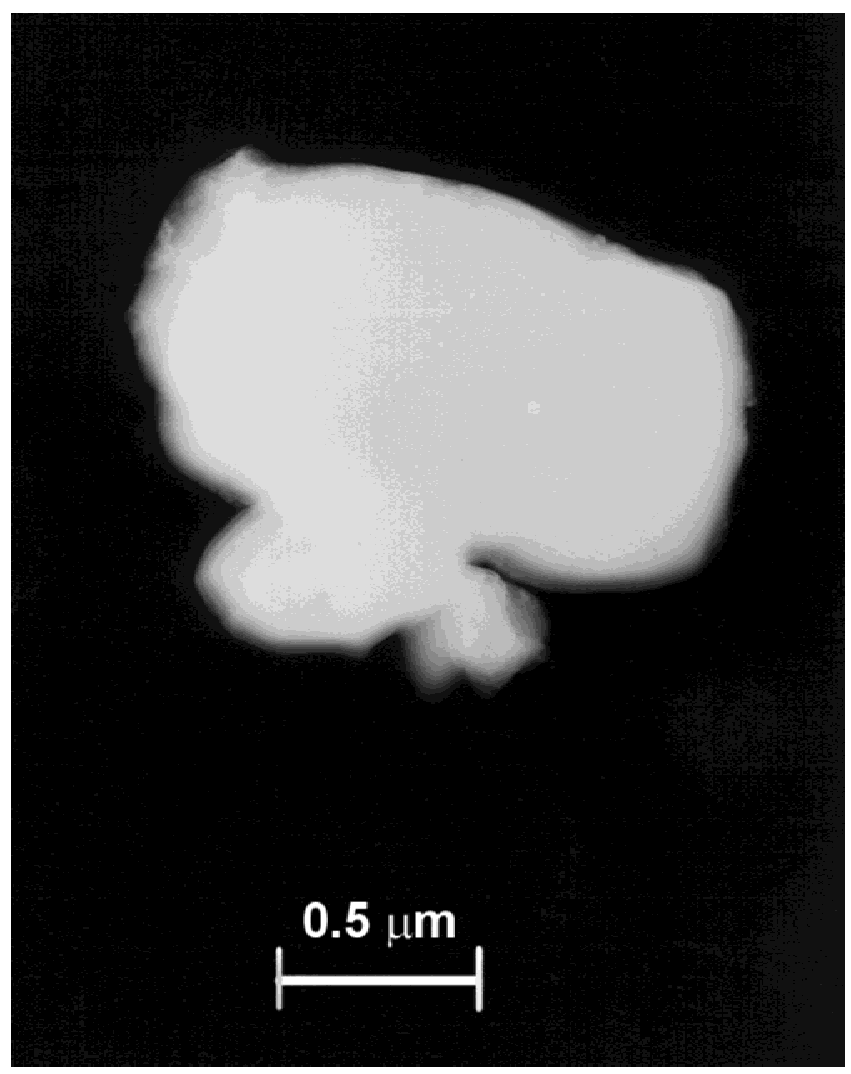

FIG. 2. TEM of BT particles.

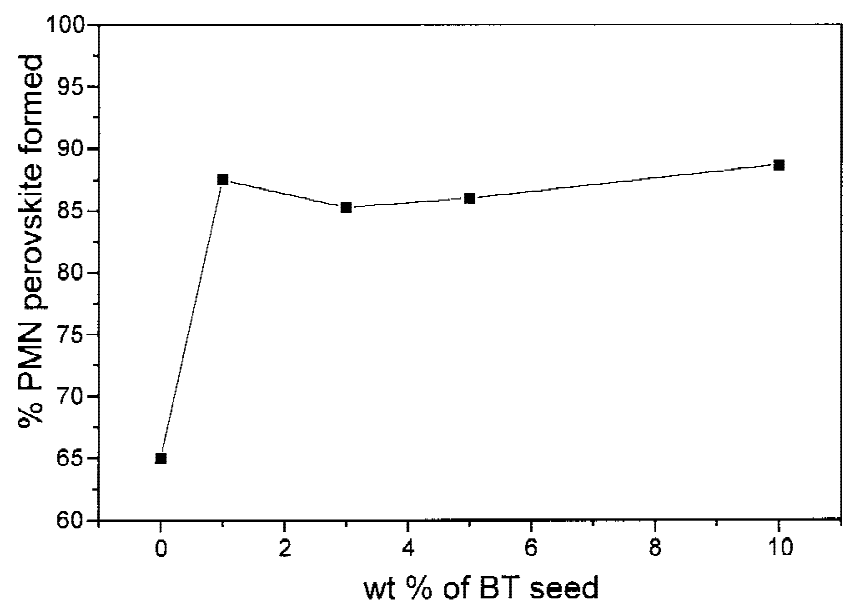

FIG. 3. Quantification of perovskite phase versus BT seed for the samples calcined at $800^{\circ} \mathrm{C}$ for $2 \mathrm{~h}$. formation of perovskite particles on the surface of this nucleus. However, compared to the results obtained by Narendar and Messing, ${ }^{20}$ the quantity of perovskite phase formation by Pechini's method is smaller (98\% with $>5.7 \mathrm{wt} \%$ BT and here $90 \%$ with $>5 \mathrm{wt} \%$ BT). This difference can be related to the complex stability originating from different precursor solution. In Narendar and Messing's study, they used ethylenediamine tetraacetic acid as a complex agent, while in this work, citric acid was used. It is known that different complex agents have different stabilities, which are directly related to perovskite phase formation. Maybe that is why there are different results in this two studies.

The obtained powder was compacted in the form of pellets and then sintered in a dilatometer furnace with a constant heating rate. The linear shrinkage in function of temperature was determined for all compositions during the sintering process. The pure PMN sample exhibits two shoulders, one around $760{ }^{\circ} \mathrm{C}$ and other around $900{ }^{\circ} \mathrm{C}$. This low-temperature shoulder is related to intraagglomerated densification process. For BT seeded samples this low-temperature shoulder appeared at higher temperatures (Figs. 4 and 5).

According to Narendar and Messing, seeding PMN with $5 \mathrm{wt} \%$ BT significantly reduces the PMN particle size and increases the amount of perovskite phase. ${ }^{20}$ These effects could reduce the temperature for the solidstate sintering of intra-agglomerates. However, the opposite occurred for the seeded samples with 1, 3, 5, and $10 \mathrm{wt} \%$ BT particles. In these cases the low-temperature shoulders appeared at $900,907,930$, and $890{ }^{\circ} \mathrm{C}$, respectively. Then the possible explanation for the sintering behavior of the intra-agglomerates could be related to the lead-rich liquid phase formation. When seed particles are present, the amount of perovskite phase is higher; consequently, the composition of the liquid phase is different

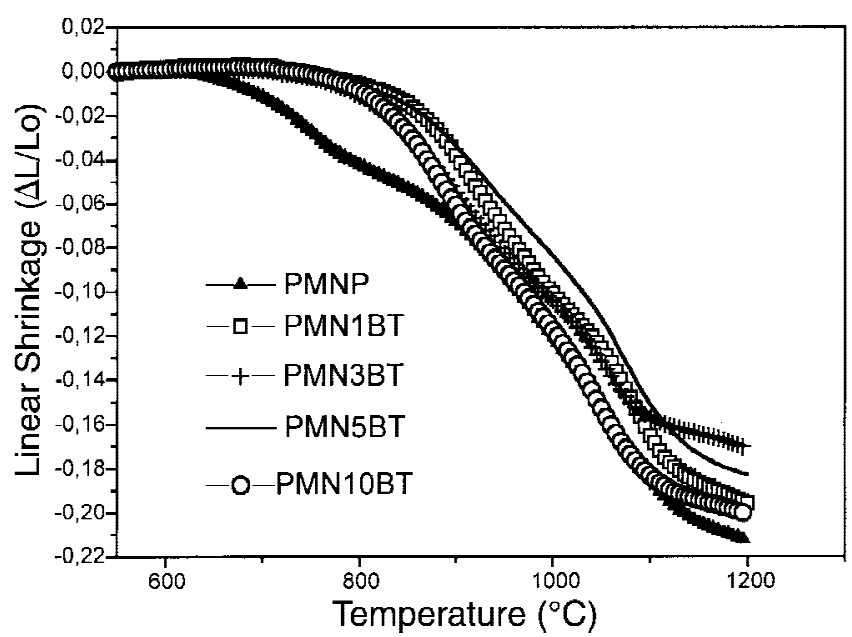

FIG. 4. Linear retraction for the pure and seeded PMN in the different compositions 
and the temperature of liquid phase formation is higher. This is in accordance with Fig. 5, where the shoulder corresponding to the sintering of intra-agglomerates is displaced to higher temperatures when seed particles are presented.

For the sintering process among the agglomerates, the highest linear shrinkage rate, occurs at 1090, 1090, 1065, 1060 , and $1045{ }^{\circ} \mathrm{C}$ respectively for pure PMN and with $1,3,5$, and $10 \mathrm{wt} \%$ BT. The linear shrinkage rate shoulder for pure PMN is broad while the shrinkage rate shoulders for seeded PMN are narrower and depend on the BT seed concentration. Considering that the PMN agglomerates decrease with the BT seed concentration, the highest shrinkage rate of seeded PMN decreases with BT seed concentration; this is shown in Fig. 6. The narrower shoulder for the BT-seeded PMN also indicates that the sintering process is more homogeneous for the seeded samples.

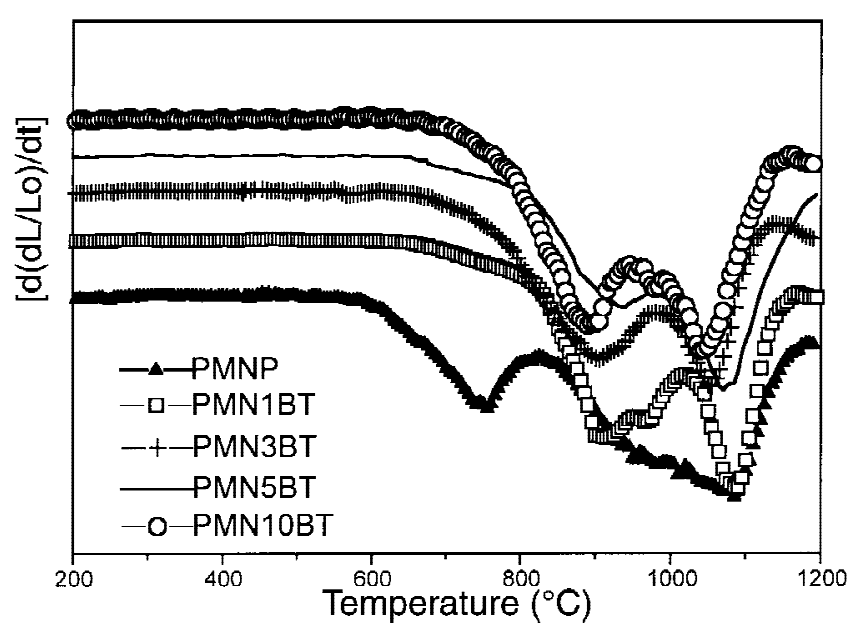

FIG. 5. Linear shrinkage rate in function of the temperature for the pure and seeded PMN samples.

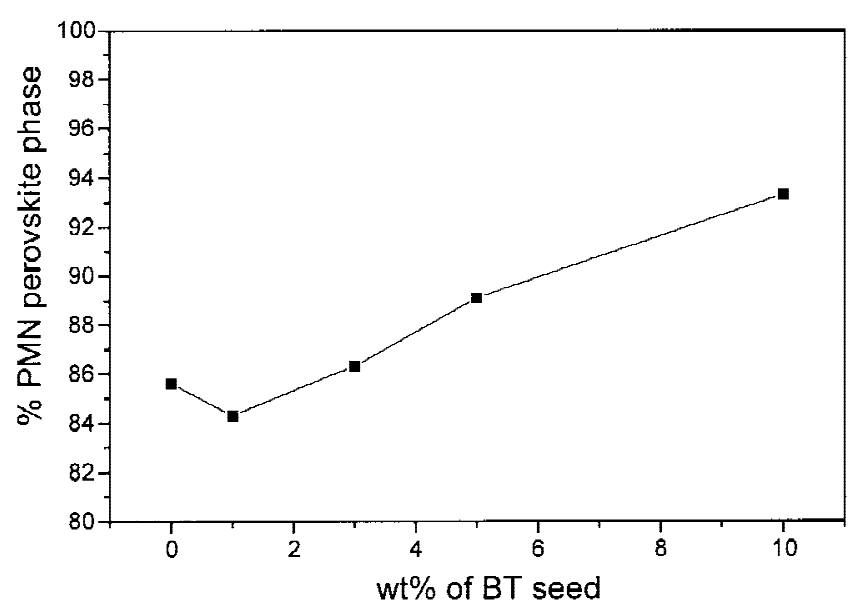

FIG. 6. Quantification of perovskite phase versus BT seed for the samples sintered at $1200{ }^{\circ} \mathrm{C}$ for $3 \mathrm{~h}$.
The PMN phases were quantified by XRD after the sintering process (Fig. 6). For the pure sample, the amount of perovskite phase visibly increased compared to the calcined sample, probably due to the higher temperature treatment. In Table I the results of the quantitative phase analysis are given together with unit cell parameter and volume for the sintered samples. The crystal structure parameters used for perovskite phase was given by the space group $P m 3 m$, and the pyrochlore phase by $F d 3 m$. In Table II the refinement indexes are given. We can observe that the amount of pyrochlore phase present in all samples, except for PMN10BT, does not change significantly as the amount of BT seeds increase, although the unit cell volume for both phases slightly decrease. These are indications that although $\mathrm{Ba}$ and $\mathrm{Ti}$ are incorporated into the Pe and Py phases, their amounts are not enough to induce the formation of Pe phase as desired. For the case with 10\% BT, the amount of Pe phase increased by $9 \%$, and this behavior can be justified mainly due to the presence of more nucleus growth. Then only the addition of $10 \%$ of isostructural seed favors the formation of the perovskite phase to the detriment of the pyrochlore one in the sintered material.

It is known ${ }^{14}$ that the ionic radius of barium $(1.61 \AA)$ is larger than the radius of ion lead $(1.49 \AA)$, which would be responsible for the increase of the volume of the cell and consequently of the lattice parameters. Supposing that only this substitution happens, the result is in agreement with that checked by Butcher and Thomas. ${ }^{15}$ Otherwise, the atomic radii of niobium $(0.64 \AA)$ and magnesium $(0.72 \AA)$ are bigger then the titanium radius $(0.605 \AA)$. Therefore, it is expected that the substitution of magnesium and niobium for titanium promotes a decrease of the unit cell sizes. ${ }^{16}$ In this work, the two substitutions occurred simultaneously during the calcining and sintering process, which supplies enough energy so that the diffusion of the barium and of the titanium happens inside of the perovskite structure as well as the pyrochlore structure; the final result is then a decrease in the unit cell. This behavior can be checked by the alteration of the lattice parameters obtained during the refinement (Table I). This behavior is in agreement with that observed in the literature..$^{5,17,18}$ Related to the increasing sintering temperature there is a diffusion of $\mathrm{Ba}$ and $\mathrm{Ti}$ atoms inside the perovskite cell as well as the pyrochlore cell, changing the lattice parameter when compared with PMN without seed. The qualitative results from the weight intensity equation is in agreement with those obtained from the refinement results. In other words, it is not exactly the same but the behavior is in agreement. To verify whether the refinement was good, it is necessary to take a look at the $R$ factors. This indexes are showed in Table II where the $R_{\mathrm{wp}}$ is quantified, which is responsible for the weighted pattern and is related to the profile. While $R_{\mathrm{B}}$ is the value for Bragg intensities and $S$ is the 
TABLE I. Lattice parameter as a function of amount of BT addition for sintered material.

\begin{tabular}{|c|c|c|c|c|c|c|}
\hline \multirow[b]{2}{*}{ Sample } & \multicolumn{3}{|c|}{ Perovskite phase } & \multicolumn{3}{|c|}{ Pyrochlore phase } \\
\hline & wt $\%$ & $a(\AA)$ & Volume & $\mathrm{wt} \%$ & $a(\AA)$ & Volume \\
\hline PMNP & $82.3(4)$ & $4.04546(4)$ & $66.207(1)$ & $17.7(.7)$ & $10.5946(3)$ & $1189.23(3)$ \\
\hline PMN1BT & $84(2)$ & $4.04485(5)$ & $66.177(1)$ & $15.9(7)$ & $10.5917(2)$ & $1188.24(3)$ \\
\hline PMN3BT & $85.4(5)$ & 4.04393 (2) & $66.132(1)$ & $14.6(7)$ & $10.58733(3)$ & $1186.747(8)$ \\
\hline PMN5BT & $85.7(2)$ & 4.04279 (2) & $66.076(1)$ & $14.3(7)$ & $10.5838(1)$ & $1185.56(3)$ \\
\hline PMN10BT & $91.7(8)$ & $4.03969(9)$ & $65.924(3)$ & $8(1)$ & $10.5746(3)$ & $1183.46(9)$ \\
\hline
\end{tabular}

TABLE II. Rietveld refinement indexes for all samples.

\begin{tabular}{lcccc}
\hline \hline Sample & $R_{\mathrm{WP}}(\%)$ & $S$ & $R_{\mathrm{B}}$ (perovskite) & $R_{\mathrm{B}}$ (pyrochlore) \\
\hline PMNP & 10.10 & 1.58 & 3.32 & 11.15 \\
PMN1BT & 10.28 & 1.66 & 3.30 & 8.96 \\
PMN3BT & 10.05 & 1.65 & 2.76 & 9.71 \\
PMN5BT & 9.79 & 1.62 & 3.20 & 10.68 \\
PMN10BT & 11.96 & 1.38 & 3.49 & 14.61 \\
\hline \hline
\end{tabular}

"goodness of fit"; and the closer it is to 1 , the better the fit. With this results it is possible to say that the refinement is acceptable.

\section{CONCLUSION}

The addition of isostructural BT seed favors the formation of the perovskite phase over the pyrochlore phase in the sintered material. The increase in the perovskite phase formation can be justified due to the presence of nucleus, which possesses a lattice parameter very similar to the desired perovskite phase, only $1 \%$ misfit, providing patterns of growth favoring the formation of perovskite particles on the surface of this nucleus. In the sintering study using a dilatometer furnace, the difference of behavior between pure and seeded sintered samples is justified due to the presence of different types of agglomerates and by the lead diffusion.

The study of the lattice parameters of the samples after the sintering process indicates that the diffusion of the titanium and of the barium inside perovskite and pyrochlore PMN phases occurs. Moreover, this substitution provoked a decrease of the lattice parameters as showed by Rietveld refinement.

\section{ACKNOWLEDGMENTS}

The authors acknowledge the São Paulo State Research Foundation (FAPESP), Brazilian National Research Council (CNPq), and Financing of Studies and Projects (FINEP/PRONEX) for the financial support of this work.

\section{REFERENCES}

1. M.A. Sekar and A. Hallyal, J. Am. Ceram. Soc. 81, 380 (1998).

2. T. Yamamoto and K. Okazaki, J. Am. Ceram. Soc. 68, C86 (1985).

3. F. Chaput and J.P. Boilot, J. Am. Ceram. Soc. 72, 1355 (1989).

4. L.F. Francis, Y.J. Oh, and D.A. Payne, J. Mater. Sci. 25, 5007 (1990).

5. J.C. Carvalho, C.O. Paiva-Santos, M.A. Zaghete, C.F. Oliveira, and J.A. Varela, J. Mater. Res. 11, 7 (1996).

6. S.L. Swartz and T.R. Shrout, Mater. Res. Bull. 17, 1245 (1982).

7. C.H. Lu and J.T. Lee, J. Ceram. Soc. Jpn. 103, 1122 (1995).

8. R.A. Shelleman and G.L. Messing, J. Am. Ceram. Soc. 71, 317 (1988).

9. M.P. Pechini, US Patent No 3330697 (1967).

10. J.O. Eckert, C.C. Hung-Houston, B.L. Gersten, M.M. Lencka, and R.E. Riman, J. Am. Ceram. Soc. 79, 2929 (1996).

11. H.M. Rietveld, J. Appl. Crystallogr. 2, 65 (1969).

12. R. Young, A. Sakthivel, T. Moss, and C.O. Paiva-Santos, J. Appl. Cryst. 28, 366 (1995).

13. J.L. Mcardle and G.L. Messing, J. Am. Ceram. Soc. 69, C98 (1986).

14. R.D. Shannon, Acta Crystallogr. A32, 751 (1976).

15. S.J. Butcher and N.W. Thomas, J. Phys. Chem. Solids 4, 595 (1991).

16. L.F. Francis and D.A. Payne, J. Am. Ceram. Soc. 74, 3000 (1991).

17. O. Sakurai, M. Katsumoto, K. Shinozaki, and N. Mizutani, J. Ceram. Soc. Jpn. 101, 594 (1993).

18. D.H. Kang and K.H. Yoon, J. Mater. Science 26, 56 (1991).

19. B.D. Begg, E.R. Vance, and J. Nowotny, J. Am. Ceram. Soc. 77, 3186 (1994).

20. Y. Narendar and G.L. Messing, J. Am. Ceram. Soc. 82, 1659 (1999). 